\title{
Present Situation of Mobile Medical Applications
}

\author{
Feng Xu, Shiqiang Zhang* \\ College of Medical Informatics, Chongqing Medical University, Chongqing, China \\ Email:904073542@qq.com, "math808543@sina.com
}

Received 14 October 2015; accepted 12 March 2016; published 15 March 2016

\begin{abstract}
Mobile medical Application (APP) is not only the product of the information age but a good helper of modern medical. In this paper, the status of mobile medical APP is described in terms of the implementation of mobile medical App, the classification of mobile medical App, mobile medical App quality and content, problems of mobile medical App.
\end{abstract}

\section{Keywords}

\section{Mobile Medical Applications, Present Situation}

March 2015, the US Food and Drug Administration (FDA) launched an Application called "Drug Shortages" to help health care doctors and pharmacists to track drug shortages, to solve the problem of drug shortages and drug production. At present, the development of mobile medical Applications is growing, and the present situation of mobile medical App is briefly described.

\section{Implementation of Mobile Medical App}

In the United States, the United States has a high demand for patient information security, a special HIPPA (Health Insurance Portability and Accountability Act), to regulate the transmission and use of information. The bill can effectively implement the management processes, information technology and other security measures to protect the patient's privacy, maintaining the integrity of patients or user related data. Part of the App in the design has strictly followed the HIPPA act to ensure the security and stability of data transmission. Mobile App store HApptique (HApptique is a program for Approving the audit of the medical APP), ensure mobile medical App access to the certification and operation of the certification of mobile medical App standard draft [1]. The draft focused on the operation of mobile health App standards, privacy standards, safety standards and content standards. In addition, different kinds of App also have the corresponding reference standard [2].

\section{Classification of Mobile Medical App}

The mobile medical App is divided into two categories by the United States FDA. One is to help users form a

${ }^{*}$ Corresponding author. 
healthy lifestyle. Another kind of App turns the role of the mobile phone to be mobile medical equipment, record blood pressure and electrocardiogram, and sent the recorded data to the doctor. Chang Liu and other [3] divided mobile medical App into 7 categories based on the function of mobile medical App. $1^{\text {st }}$, medical information reference class, this class of App is basically from the paper version of the conversion, such as ICD-9, diabetes prevention and treatment guidelines, coronary heart disease treatment. $2^{\text {nd }}$, drug and medical information database. This class of App to provide information on disease and drug information inquiries. $3^{\text {rd }}$, decision support class, this class of App provides information or data support for the clinical decision of a doctor. $4^{\text {th }}$, medical counting class, such as medical calculator. $5^{\text {th }}$, tracking tool, this class of App records the patient's signs data, such as blood sugar, blood pressure, heart rate, etc. $6^{\text {th }}$, educational tools, this kind of App is mainly aimed at continuing education of doctors or medical students, such as medical English Dictionary. $7^{\text {th }}$, other classes, such as oral health, vision testing, weight management, etc.

\section{Mobile medical App Regulations}

The presence of medical industry risks leading to the development of mobile medical App in a variety of challenges ahead, so the security and supervision of App is particularly important. In June 2009, the Swedish Medical Equipment Association pointed out that the use of independent, personal diagnosis and health care and treatment to produce direct or indirect impact of the software should be subject to the supervision of product safety regulations. At the same time, the association also pointed out that the use of medical purposes should be used as conventional equipment, and to accept the legal supervision of the European medical equipment [4]. The American Medical Informatics Association filed a regulatory opinion on mobile health App in 2011, including the definition, classification, function, use of the term, and the assessment of the delivery and payment of medical services to patients with App [5]. In July 2011, the US promulgated the "mobile device medical class App Management Act", the first of the disease prevention, diagnosis, treatment and other functions of App into the regulatory scope. In September 2013, the United States FDA officially released the mobile medical App regulatory documents. The mobile medical App has been strictly defined, and determines what App should be included in the regulatory range of [6].

\section{Mobile Medical App Quality and Content}

BA Rosser and other [7] studied 4 operating systems, a total of 111 App about management of pain. The results show that the content of this kind of App is not accurate enough because of the risk of misleading patients. Bierbrier $\mathrm{R}$ and others [8] analyzed the accuracy of the 13 functions of the 14 App. The results show that the overall accuracy of the App is $98.6 \%$, and the accuracy of 6 App of these 13 functions is as high as $100 \%$. Demidowich and others [9] evaluated 6 App from 42 diabetes management from the Android system. The average score of availability of these Apps was 11.3. The results show that almost no App can provide a better way to manage chronic disease. Tousignant-Laflamme Yand others [10] was used to analyze the internal and external reliability of two App. The results show that the two App's internal reliability is general, external reliability is low. Katy Mitchel and others [11] discussed the validity and reliability of the two shoulder rotation measurement App named Dr. Goniometry and GetMyROM. It is found that the reliability and validity of these two Apps are relatively high.

\section{Problems of Mobile Medical App}

\subsection{Classification Confusion}

Mobile medical App has a clear definition in the United States, and FDA delineated the scope of regulation. Despite the presence of China Mobile Medical Special Committee, the committee did not explicitly pointed out that the related concepts of mobile medical and mobile medical. In addition, App lack of strict classification standards, the majority of health information App even the entertainment game App is also classified as medical class, and these App actually does not have any medical function.

\subsection{Low Access Threshold and a Lack of Regulatory Mechanisms}

Mobile medical App is a very low threshold for the mobile medical industry entrance, so the increase in App is 
rapid. But health care is a very special industry, professional and strong, great risk, a variety of disputes. It is necessary to accept strict supervision. If only for the sake of App more easily be used and deliberately bypass the regulatory link, then the App is not professional or even wrong information on the user has a greater security risks, and even life-threatening health. Thus the value of mobile medical App does no longer exist. Most entrepreneurs of the domestic mobile medical App field were from the information technology industry, not the medical industry. Market lack of professional barriers and access to App companies and the audit mechanism, and even a large part of the App is simply transformed the paper into electronic version, such as the "Treatment of coronary heart disease”. In addition, the mobile medical industry is lack of relevant laws and regulations or industry standards to manage, and there is no authority to audit institutions. Whether the content of the App content in the medical industry is not known and the content of App is poor.

\subsection{Low Privacy and Low User Trust}

Mobile medical App operation based on wireless network, once the malicious attack or error operation may reveal the user's personal information. The vast majority of mobile medical App does not have the privacy protection function, the user's information security is not guaranteed, easy to be leaked or stolen. Mobile health App audit lack of scientific and evaluation criteria standardized, so now the mobile health App have a lot of cheating and cohabitation. In terms of information sources, a lot of mobile medical App, there is no source of the original information, nor does it show that the reference guide and magazine. The vast majority of App did not strictly follow the clinical guidelines. Evidence-based medicine based on the development of App, the reliability is not high. Some of the App' questions come from Internet search or other question and answer platform, such as Baidu. People who answers questions may be any one of the users, most likely not have any medical knowledge or misleading the other users. Credibility is very low, which leads the user to lose confidence in App. For the user, the authenticity and reliability of online question answering doctor's identity is also questioned.

\section{Acknowledgement}

Thanks to my tutor Professor Zhang's guidance on the paper. And thank him for his help and guidance on my life. Thanks for my college and university.

\section{References}

[1] Nicole Lewis. Mobile Health App See Weak Adoption Rates. http://news.hc3i.cn/art/201011/8222.htm

[2] Salditt (2004) Trends in Medical Device Design and Manufacturing. Journal of SMT, 17, 19-22.

[3] Liu, C., Zhu, Q., Kenneth, A., et al. (2011) Status and trends of mobile-health Applications for iOS devices A developer s perspective. The Journal of System and Software, 84, 2022-2033. http://dx.doi.org/10.1016/j.jss.2011.06.049

[4] Medical Products Agency (2010) Project Summary Document. The Medical Products Agency’s Working Group on Medical Information Systems.

[5] AMIA Comments on FDA Mobile Medical App Draft Guidance.

[6] US Department of Health and Human Services Food and Drug Administration. Draft Guidance for Industry and Food and Drug Administration Staff-Mobile Medical Applications. Food and Drug Administration, USA, 2013.

[7] Rosser, B.A. and Ecclestone, C. (2011) Smartphone Applications for Pain Management. Journal of Telemedicine and Telecare, 17, 308-312. http://dx.doi.org/10.1258/jtt.2011.101102

[8] Bierbrier, R., Lo, V., Wu, R.C., et al. (2014) Evaluation of the Accuracy of Smart Phone Medical Calculation App. Journal of Medical Internet Research, 16, e32.

[9] Demidowich, A.P., Lu, K., Tamler, R. and Bloomgarden, Z. (2012) An Evaluation of Diabetes Self-Management Applications for Android Smartphones. Journal of Telemedicine and Telecare, 18, 235-238. http://dx.doi.org/10.1258/jtt.2012.111002

[10] Tousignant-Laflamme, Y., Boutin, N., Dion, A.M., et al. (2013) Reliability and Criterion Validity of Two Applications of the iPhone to Measure Cervical Range of Motion in Healthy Participants. Journal of NeuroEngineering and Rehabilitation, 10, 69-78. http://dx.doi.org/10.1186/1743-0003-10-69

[11] Mitchell, K., Gutierrez, S.B., Sutton, S., et al. (2014) Reliability and Validity of Goniometric iPhone Applications for the Assessment of Active Should Externalrotation. Physiotherapy Theory and Practice [Epub Ahead of Print]. http://dx.doi.org/10.3109/09593985.2014.900593 